\title{
The Development of Risk Index Algorithm Which is applied to Chemical Accident Behaviour Analysis/Damage Prediction Models and Environmental Risk Map Technology
}

\author{
Ji-Hoon Seo, Yoon Jae Kim, Jong-Ryeul Sohn \\ Korea University \\ 145, Anam-ro, Seongbuk-gu, Seoul, Republic of Korea \\ Wlgns0220@naver.com
}

\section{Extended Abstract}

The increasing risk and complexity of chemicals being produced and transported has resulted in more rigorous demands on both people as well as chemical-handling industries to manage the risks involved. The Ministry of Environment has been carrying out the Korean National Environmental Health Survey to systematically and continuously investigate the influencing factors of people's level of exposure to hazardous pollutants and use the data as basic information of environmental health policies.

We had been proposed a plan of Industry-university collaborative technology development projects at which make environmental risk map appear on the GIS through linkage between Risk index and behaviour analysis/damage prediction model. The one of the study is development of risk index development methodology/process which is a planning tool created through literature survey and experts review and advice about indices related to the EHS field.

In this study, we reviewed various indicators which had been listed to calculate a risk index. And based on this, Algorithm was completed. To increase the effectiveness, we derived limitations and supplement point. This study is considered to be exploited new risk index through the development of methodology and process, as the basis for the chemical incident response and environmental impact prediction technology.

\section{References}

[1] S. Asa. "Environmental-accident index: validation of a model," Journal of Hazadous Materials, vol. 61, pp. 305-312, 1998. 